\title{
EMPATIA EM SAÚDE: REVISÃO INTEGRATIVA
}

\section{EMPATHY IN HEALTH: INTEGRATIVE REVIEW \\ EMPATÍA EN SALUD: REVISIÓN INTEGRADORA}

Leandro Felipe Mufato ${ }^{1}$, Maria Aparecida Munhoz Gaíva ${ }^{2}$.

\section{RESUMO}

Objetivo: Analisar as publicações de pesquisas disponíveis na literatura sobre empatia no cuidado em saúde. Método: Revisão integrativa com busca de estudos primários realizada nas bases de dados LILACS, PSYCINFO, WEB OF SCIENCE, BDENF, MEDLINE e CINAHL, sem restrição de período e idioma. Resultado: A análise de 33 estudos primários mostra publicações de 1968 a 2016 , originárias de diferentes regiões do mundo. Há predomínio da abordagem quantitativa com o uso de diferentes escalas psicométricas autoaplicáveis para mensurar os níveis de empatia de profissionais da saúde, estudantes ou pacientes. Há diversidade nas populações pesquisadas, locais de estudo e falta consenso entre os autores sobre como a empatia se desenvolve, perpassando explicações pelos campos da cognição, emoção e atividade neuromotora. Conclusão: O debate sobre a empatia está em pleno desenvolvimento, especialmente, quando se observam as diferentes explicações para sua ocorrência. A empatia é um forte constructo capaz de contribuir para pesquisas sobre a relação profissional-paciente.

Descritores: Empatia; Relações profissional-paciente; Enfermagem; Relações médico-paciente; Revisão.

\section{ABSTRACT}

Objective: To analyze the research publications available in the literature related to empathy in health and nursing care. Method: Integrative revision, searching for the primary studies was carried out in the LILACS, PSYCINFO, WEB OF SCIENCE, BDENF, MEDLINE and CINAHL databases, without period and language restriction. Results: The analysis of 33 primary studies shows publications from 1968 to 2016, from different regions of the world. There is a predominance of the quantitative approach, with the use of different self-applicable psychometric scales to measure the levels of empathy of professionals, health students or patients. There are diversities in the surveyed populations and places of study, with lack of consensus among authors on how empathy develops, proceeding explanations fields of cognition, emotion and neuro-motor activity. Conclusion: The debate on empathy is in full development, especially when we observe the different explanations for its occurrence. Empathy is a strong construct capable of contributing to research on the professional-patient relationship.

Descriptors: Empathy; Professional-patient relations; Nursing; Physician-patient relations; Review.

\section{RESUMEN}

Objetivo: Analizar las publicaciones de investigación disponibles en la literatura sobre la empatía en la atención en salud y enfermería. Métodos: Revisión integradora con búsqueda de los estudios primarios en las bases de datos LILACS, PSYCINFO, WEB OF SCIENCE, BDENF, MEDLINE y CINAHL, sin restricción de tiempo y lenguaje. Resultados: El análisis de 33 estudios primarios demuestra publicaciones entre 1968-2016, de diferentes regiones del mundo. Hay un predominio del enfoque cuantitativo, utilizando escalas psicométricas auto-a8dministradas para medir níveles de empatía de profesionales, estudiantes o pacientes. Hay diversidad en las poblaciones estudiadas, los sitios de estudios y la falta de consenso entre los autores sobre cómo se desarrolla la empatía, que corre a través de diferentes explicaciones. Conclusión: El debate sobre empatía está en pleno desarrollo, especialmente al observar las diferentes explicaciones para su ocurrencia. La empatía es un constructo teórico, capaz de contribuir a la investigación sobre la relación profesional-paciente.

Descriptores: Empatía; Relaciones profesional-paciente; Enfermería; Relaciones médico-paciente; Revisión.

${ }^{1}$ Professor e pesquisador da Universidade do Estado de Mato Grosso. ${ }^{2}$ Doutora. Orientadora no Programa de Pós-Graduação em Enfermagem da UFMT. 


\section{INTRODUÇÃO}

A empatia é considerada um elemento central nas relações entre profissionais da saúde e seus pacientes, trazendo benefícios para ambos. Pode ser entendida como um dos elementos que contribui para a melhor compreensão dos aspectos dinâmicos das interações sociais em saúde. O cuidado empático envolve a valorização da equidade em saúde, com sensibilização recíproca entre profissional e paciente, e do reconhecimento do outro em sua singularidade e complexidade. Assim, necessário para as profissões da área da saúde ${ }^{(1)}$.

No Brasil, o modo como os profissionais de saúde interagem com os pacientes tornou-se ainda mais relevante, com a promulgação da Política Nacional de Humanização ${ }^{(2)}$, um marco na luta contra $O$ atendimento desumanizado, estritamente biomédico e despersonalizado que a população recebia nos serviços de saúde brasileiros. Ainda, atender aos sujeitos de modo integral e equânime, princípios do Sistema Único de Saúde, tem exigido uma mudança atitudinal e de comportamento dos atores sociais aí envolvidos $^{(3)}$. O contexto brasileiro de investimento em humanização sustenta o interesse sobre a empatia nos cenários de atendimentos de saúde, tendo em vista que a principal insatisfação que se quer combater com a proposta da humanização é sobre os aspectos de relacionamentos com os profissionais de saúde ${ }^{(4)}$. Mesmo assim, a implantação de propostas de cuidados humanizados, ainda não é uma realidade de todos os serviços de saúde, o que demanda maior discussão sobre esse aspecto do cuidado em saúde ${ }^{(5-6)}$.

$\mathrm{Na}$ questão relacional entre profissional e paciente, já se sabe que a empatia dos médicos possibilita maior satisfação dos pacientes e amplia a habilidade para diagnósticos, tratamento, e diminui o risco de erros médicos; a empatia aumenta a aceitação e sensação de pertencimento dos pacientes e melhora a relação entre profissionais e pacientes pediátricos oncológicos, acrescendo a resiliência destes ao sofrimento ${ }^{(7-9)}$.

Ademais, alguns estudos demonstram a associação entre bons resultados clínicos e a empatia, tais como melhor percepção das necessidades de saúde, redução da ansiedade e estresse psicológico ${ }^{(8,10-12)}$. Na psicologia, área que trouxe o conceito da filosofia para o campo científico, ela é essencial em muitas linhas de tratamento.
Mesmo a empatia sendo uma característica central da interação entre profissionais da saúde e pacientes, seus baixos níveis nos profissionais são documentados e discutidos na literatura científica $^{(12)}$. Ademais, materializar o comportamento empático é um desafio no cotidiano das instituições de saúde ${ }^{(13)}$.

A empatia é um conceito polissêmico que aponta para várias frentes de entendimento sobre sua natureza, não havendo ainda um consenso científico ${ }^{(14)}$. Esta pesquisa, visa a contribuir para termos uma visão mais clara da extensão, alcance e natureza da atividade de pesquisa em torno da empatia. Busca sumarizar e disseminar os resultados de pesquisa já produzidas, bem como, identificar as lacunas para investimentos em pesquisas futuras.

A relevância da presente pesquisa consiste na síntese do conhecimento já produzido por pesquisas sobre a empatia na saúde. Os resultados podem trazer benefícios ao campo das investigações científicas sobre a relação profissional-paciente, contribuindo para futuras pesquisas sobre o tema. Dessa forma, essa investigação foi desenvolvida para responder ao seguinte questionamento: Como se caracteriza a produção científica sobre a empatia em saúde? Este estudo tem como objetivo analisar as publicações de pesquisas disponíveis na literatura sobre a empatia em saúde.

\section{MÉTODO}

Estudo de Revisão Integrativa (RI) da literatura, que seguiu as seguintes etapas: identificação da questão de pesquisa; busca na literatura dos estudos primários; avaliação dos dados; análise; síntese dos dados; e, apresentação dos resultados ${ }^{(15)}$. A questão que norteou esta revisão integrativa foi: Como se caracteriza a produção científica sobre a empatia em saúde?

As bases de dados selecionadas foram LILACS, PSYCINFO, WEB OF SCIENCE, BDENF, MEDLINE e CINAHL. Tais bases foram eleitas pela quantidade de indexação de artigos na área da saúde; por divulgarem estudos primários de temas direcionados à saúde.

Os descritores foram escolhidos de acordo com a população, o fenômeno e o contexto investigado. Para o levantamento dos artigos, utilizaram-se os descritores "profissionais da saúde" e "empatia". O termo "empático" e "cuidado em saúde" foi utilizado nas combinações como termo chave, mesmo não 
sendo um descritor previsto pelo DeCS ou MESH, pois as buscas iniciais demonstraram que os termos permitiam um levantamento mais sensível ao tema. A maior sensibilidade da estratégia de busca, maior número de estudos recuperados por estratégia de busca ${ }^{(16)}$, é bemvinda nesta pesquisa, que procura mapear de modo amplo o que se produziu sobre empatia em saúde. O cruzamento desses descritores nas bases citadas ocorreu por meio do operador booleano AND (profissionais da saúde AND empatia; profissionais da saúde AND empático; cuidado em saúde AND empatia; cuidado em saúde AND empático).

Os critérios de inclusão utilizados foram: publicação de resultados de pesquisa com uso de dados primários; tendo como foco de investigação (objeto de estudo) a empatia de profissionais no contexto do cuidado em saúde, seja na formação ou na atuação profissional. Como exclusão, optou-se por retirar os ensaios teóricos e notas editoriais, por se tratar de opiniões de especialistas sem coleta de dados primários. Não houve delimitação do idioma e nem do período de publicação.

Para confirmar se o estudo atendia aos critérios propostos e demonstrar o fluxo de seleção dos dados, foram aplicadas as etapas propostas pelo PRISMA Statement ${ }^{(17)}$, no qual, após identificados os registros nas bases de dados e retiradas as duplicações, foram lidos títulos e resumos para rastrear estudos pertinentes. Em seguida, foi realizada a leitura na íntegra do material para a elegibilidade, com posterior síntese dos estudos incluídos.

A coleta de dados ocorreu durante o período de abril a agosto de 2016. Para busca de textos não disponibilizados na íntegra, recorreu-se à procura deles no Portal de Periódico da Coordenação de Aperfeiçoamento de Pessoal de Nível Superior (Periódico CAPES). Na Figura 1, mostra-se o caminho percorrido para seleção das publicações, de acordo com os critérios de inclusão e exclusão, com uso do fluxograma PRISMA.

Figura 1 - Fluxograma de seleção de artigos elaborado com base no PRISMA.

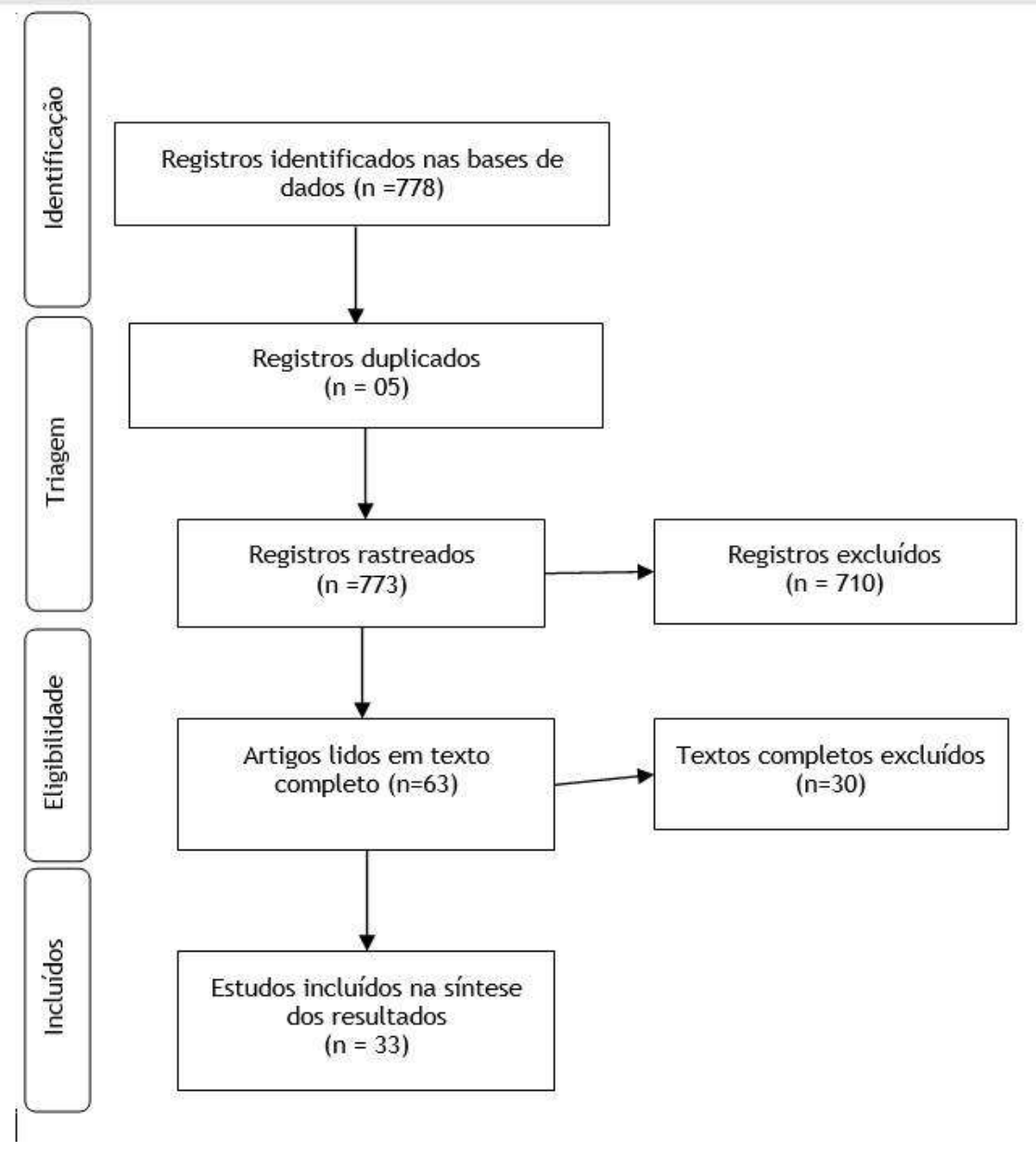

Fontes:

Fluxograma: Declaração PRISMA. Dados: bases de dados LILACS, PSYCINFO, MEDLINE e CINAHL. 
Nas bases de dados, as estratégias utilizadas resultaram em 778 publicações, distribuídas na seguinte quantidade: 415 (53,34\%) na Medline; 206 (26,48\%) na Cinahl; 101 (12,99\%) na Psycinfo; 37 (4,75\%) na Web of Scicence; Lilacs, 15 (1,93\%); e, BDENF, 04 $(0,51 \%)$. Com o refinamento, aplicação dos critérios de inclusão e exclusão, obtiveram-se 33 publicações.

Todas as 33 publicações se caracterizaram como artigos de periódicos científicos que divulgavam resultados de pesquisas com uso de dados primários. As bases BDENF e Web of Science apresentaram registros, porém na etapa de avaliação, seus resultados não foram elegíveis.

Após avaliação e seleção encontradas, foi realizada a análise crítica dos textos. Os dados discursivos, tais como objetivos e resultados, foram analisados e interpretados tendo como perspectiva atingir o objetivo desta pesquisa. A análise culminou com a criação de três categorias a serem apresentadas: Caracterização dos estudos sobre empatia em saúde; Métodos empregados nos estudos sobre empatia em saúde; Resultados obtidos nos estudos sobre empatia em saúde.

\section{RESULTADOS E DISCUSSÃO}

\section{Caracterização dos estudos sobre empatia em saúde}

De acordo com cada base de dados, os 33 artigos científicos sobre empatia em saúde resultantes se distribuídos da seguinte forma: 15 (45,45\%) na CINAHL; 11 (33,33\%) na Psylnfo; 06 (18,18\%) na MEDLINE; e, 1 (3,03\%) no LILACS. A primeira publicação encontrada na busca é de 1968, contudo, os últimos dez anos concentram
$14(42,42 \%)$ das produções analisadas. As publicações distribuem-se nas seguintes áreas: 12 $(36,36 \%)$ em psicologia, 11 (33,33\%) em medicina, 09 (27,27\%) em enfermagem, e 01 $(3,03 \%)$ em neurociências. Dos 33 estudos, apenas um foi publicado no idioma espanhol e os demais em inglês.

A psicologia tem avançado nas propostas sobre o funcionamento da empatia, colocando-a como uma importante habilidade de compreensão dos outros num contexto social, porém, mesmo a psicologia não possui um conceito unívoco sobre o que é a empatia ${ }^{(18-19)}$.

Apesar deste contexto, a psicologia é uma área de conhecimento que possui discussão relevante sobre o tema. Medidas de empatia em psicologia são usadas até para a escolha e seleção de profissionais em alguns países, como os Estados Unidos da América (EUA). Ademais, as produções sobre empatia em psicologia demonstram o campo como vanguarda das discussões científicas sobre o tema, não somente pelas produções mais antigas, mas, também, pela realização de estudos que apontam a empatia como componente fundamental do sucesso terapêutico na área.

A eficácia terapêutica é relacionada de várias formas ao comportamento empático do profissional psicólogo, ao passo que vários estudos testam e questionam essa eficácia comparando o comportamento empático com outras abordagens em psicologia, como demonstrado nos estudos aqui analisados ${ }^{(18,20-28)}$.

Na Figura 2, apresenta-se a caracterização dos estudos quanto aos autores, ano de publicação, local do estudo, base de dados de indexação e área da saúde.

Figura 2 - Quadro síntese com os artigos sobre empatia em saúde, segundo autores, ano de publicação, local do estudo, base de dados de indexação e área da saúde, Cuiabá, Mato Grosso, Brasil, 2017.

\begin{tabular}{|llllll|}
\hline \multicolumn{1}{|c}{ No } & Antores & Ano & Local do estudo (país) & $\begin{array}{c}\text { Base de } \\
\text { dados }\end{array}$ & Área \\
\hline 1. & Payne; Gralinski & 1968 & Estados Unidos da América & PSYCINFO & Psicologia \\
\hline 2. & Fish & 1970 & Estados Unidos da América & PSYCINFO & Psicologia \\
\hline 3. & Bachrach; Mintz; Luborsky & 1971 & Estados Unidos da América & PSYCINFO & Psicologia \\
\hline 4. & Kurtz; Grummon & 1972 & Estados Unidos da América & PSYCINFO & Psicologia \\
\hline 5. & Payne; Weiss; Kapp & 1972 & Estados Unidos da América & PSYCINFO & Psicologia \\
\hline 6. & Dalton; Sundblad & 1973 & Estados Unidos da América & PSYCINFO & Psicologia \\
\hline 7. & Heck; Davis & 1973 & Estados Unidos da América & PSYCINFO & Psicologia \\
\hline 8. & Perry & 1975 & Estados Unidos da América & PSYCINFO & Psicologia \\
\hline 9. & Kimberlin; Friesen & 1977 & Estados Unidos da América & PSYCINFO & Psicologia \\
\hline 10. & Esse; Wilkins & 1978 & Estados Unidos da América & PSYCINFO & Psicologia \\
\hline 11. & Dooley; Lange; Whiteley & 1979 & Estados Unidos da América & PSYCINFO & Psicologia \\
\hline 12. & Fernald; Corry & 1981 & Estados Unidos da América & CINAHL & Medicina \\
\hline
\end{tabular}




\begin{tabular}{|llllll|}
\hline 13. & Clay & 1984 & Inglaterra & CINAHL & Enfermagem \\
\hline 14. & Kuremyr et al. & 1994 & Suécia & CINAHL & Enfermagem \\
\hline 15 & Sterling; Friedman & 1996 & Estados Unidos da América & CINAHL & Medicina \\
\hline 16. & Nagano & 2000 & Japão & CINAHL & Enfermagem \\
\hline 17. & Bylund; Makoul & 2002 & Estados Unidos da América & CINAHL & Medicina \\
\hline 18. & Lauder et al & 2002 & Escócia & CINAHL & Enfermagem \\
\hline 19. & Beddoe; Murphy & 2004 & Estados Unidos da América & CINAHL & Enfermagem \\
\hline 20. & Stephen; Stanley; Charon. & 2008 & Estados Unidos da América & MEDLINE & Medicina \\
\hline 21. & Nicolai; Demmel; Farsch & 2010 & Estados Unidos da América & CINAHL & Medicina \\
\hline 22. & Bird et al. & 2010 & Suíça & CINAHL & Neurociências \\
\hline 23. & Hojat et al. & 2011 & Estados Unidos da América & MEDLINE & Medicina \\
\hline 24. & Soltner et al. & 2011 & França & MEDLINE & Medicina \\
\hline 25. & Eide, Sibbern; Johannessen & 2011 & Noruega & CINAHL & Enfermagem \\
\hline 26. & Wittenberg-Lyles et al. & 2012 & Estados Unidos da América & MEDLINE & Medicina \\
\hline 27. & Birhanu et al. & 2012 & Etiópia & MEDLINE & Medicina \\
\hline 28. & Kondo et al. & 2013 & Japão & CINAHL & Psicologia \\
\hline 29. & Narvaéz et al. & 2014 & Colômbia e República Dominicana & LILACS & Medicina \\
\hline 30. & Özakgül et al. & 2014 & Turquia & CINAHL & Enfermagem \\
\hline 31. & Bikker et al. & 2015 & Escócia & MEDLINE & Enfermagem \\
\hline 32. & Díaz et al. & 2015 & Estados Unidos da América & CINAHL & Enfermagem \\
\hline 33. & Seehausen et al. & 2016 & Alemanha & CINAHL & Medicina \\
\hline
\end{tabular}

Fonte: Bases de dados LILACS, PSYCINFO, MEDLINE e CINAHL.

O aumento das publicações sobre empatia, ao longo dos últimos dez anos, evidencia uma preocupação atual dos pesquisadores com a empatia no campo da saúde. Esse aumento marca também o avanço que áreas como a medicina e a enfermagem têm feito na investigação do tema, situado fortemente no campo da psicologia até a década de 1970.

$O$ fato de termos publicações sobre empatia no mundo todo, ratifica a universalidade da temática e a preocupação dos cientistas com os aspectos relacionais entre profissionais e pacientes. $E$, a variabilidade dos objetivos em pesquisas sobre empatia em saúde reforça a diversidade das frentes de investigações, mostrando que a empatia tem sido preocupação em diferentes cenários de atuação no campo da saúde.

A seleção dos estudos demonstrou que "empatia" é um termo, frequentemente, associado ao cuidado e tratamento em saúde, porém, não é tomada como objeto específico de estudo em muitos casos. Ou seja, o termo aparece em diversas pesquisas, porém, essas pesquisas tratam de outros temas que não a empatia, tais como a "humanização da assistência", no Brasil. Ao mesmo tempo em que se evidencia uma preocupação mundial com a empatia na saúde, observou-se a ausência de estudos brasileiros sobre o tema.

Talvez isso se dê pelo fato de que, no país, os aspectos relacionais entre profissionais e pacientes sejam tratados sob a ótica de outros conceitos operacionais, tais como o acolhimento, a humanização, comunicação terapêutica, entre outros.

Quanto aos objetivos das pesquisas sobre empatia em saúde, ressalta-se que $07(21,21 \%)$ delas buscam testar, validar ou comparar escalas de testes psicométricos para mensurar os níveis de empatia dos profissionais e/ou estudantes na saúde, correspondendo a maior parte dos objetivos dos estudos aqui analisados.

Demais estudos têm por objetivo a identificação e descrição da relação entre a variável "níveis de empatia" com outras variáveis, tais como burnout e características pessoais do profissional, ou resultados clínicos, como diminuição da ansiedade e adesão do paciente ao tratamento.

Há ainda, pesquisas que buscam identificar diferenças entre escalas psicométricas de empatia aplicadas a uma mesma população de estudo, ou comparar o efeito terapêutico do comportamento empático em relação às outras técnicas.

Sobre os conceitos de empatia empregados nas pesquisas, estes expressam uma complexa rede de significados. Em linhas gerais, a empatia se dá: como um atributo cognitivo, como um atributo emocional, emocional-cognitivo e neuromotor. Destaca-se que $14(42,4 \%)$ dos estudos não explicitaram o conceito de empatia usado.

Entre os estudos analisados, há os que a definem como um atributo predominantemente cognitivo do profissional. A empatia envolveria a compreensão da experiência, preocupação e 
perspectiva do paciente, combinada com a capacidade de comunicar essa compreensão(29). Outros estudos conceituaram empatia como a percepção da estrutura de referências internas de outra pessoa, com seus componentes emocionais e significados, como se fosse a outra pessoa, mas sem nunca perder a condição de "como se fosse" ${ }^{(30)}$. Na enfermagem, a empatia é conceituada como colocar a si mesmo, mentalmente e emocionalmente, no mundo do paciente, comunicar esse entendimento a ele e observar a sua compreensão sobre isso ${ }^{(31-32)}$.

O conceito de empatia tido como cognitivo-emocional considera a empatia um processo cognitivo e afetivo pelo qual é possível "saber" os sentimentos e pensamentos de outra pessoa ${ }^{(12)}$. Já a empatia como atividade neuromotora é a tendência de, automaticamente, mimetizar e sincronizar expressões faciais, vozes, posturas e movimentos de uma outra pessoa ${ }^{(33-34)}$.

Observou-se a falta de um conceito operacional comum de empatia às áreas da saúde, aceito pela comunidade acadêmica de forma consensual. Não há um consenso científico sobre os processos básicos e componentes fundamentais dela ${ }^{(14)}$. É considerável o número de pesquisadores que utilizam o conceito de empatia com base na leitura do psicólogo Carl Rogers, um dos pioneiros a discorrer sobre a importância dela para o tratamento dos pacientes na psicologia. Seu conceito é um dos mais importantes no campo da psicoterapia ${ }^{(14,30)}$.

Os autores que defendem a empatia como atributo cognitivo o fazem pressupondo que ela poderia ser ensinada e treinada nos profissionais da saúde ${ }^{(7,29,34)}$. Além disso, esses autores alertam para os perigos de se atribuir à empatia um atributo predominantemente emocional em decorrência da tensão que as emoções geram para os profissionais de saúde que lidam com situações de vida, sofrimento e morte no cotidiano do trabalho ${ }^{(29,34)}$.

Os conceitos de empatia acabam conflitando sob sua natureza, cognitiva, emocional ou neuromotora, dificultando a elaboração de um conceito operacional. Estudos precisariam ser desenvolvidos para que se entendesse como esses diferentes aspectos se relacionam e como podem ser treinados em estudantes e profissionais da saúde, haja vista ser possível incrementar as habilidades empáticas cognitivas, afetivas e comportamentais, concomitantemente, a depender do treinamento empregado ${ }^{(13)}$.

Algumas explicações sobre empatia já buscam integrar esses elementos, confirmando a participação genética que garante a estrutura cerebral para que o ser humano seja empático, mas assinalando que seu desenvolvimento sofre influência da vida social. Essas explicações tentam estudar os elementos cognitivos, emocionais e neuronais da empatia como complementares, e não como componentes antagônicos que não interagem entre $\mathrm{si}^{(14)}$.

No presente estudo de revisão, poucos autores trazem a complementariedade entre os elementos cognitivo, afetivo, comportamental e neuronal da empatia, o que pode ser explicado pelo posicionamento epistemológico já demarcado dos que defendem apenas um dos aspectos que explicam a empatia, ou pelo fato de que os estudos que buscam integrar esses elementos sejam mais recentes, apresentando uma nova vertente de discussão sobre o fenômeno complexo da empatia, ainda pouco referenciado. Ademais, outro aspecto pouco discutido na literatura é a relação do desenvolvimento moral dos profissionais em torno de uma ética do cuidado que propicie a empatia, que pode, neste âmbito, ser considerada como fonte de tratamento singular e equânime em saúde ${ }^{(1)}$.

\section{Métodos empregados nos estudos sobre empatia em saúde}

A abordagem em pesquisa predominante é a quantitativa, em $29(87,87 \%)$ das publicações, seguida da abordagem mista (integração de dados quantitativos e qualitativos) utilizada em $03(9,1 \%)$ dos estudos. A abordagem qualitativa foi empregada em apenas $01(3,03 \%)$ das investigações.

A predominância dos métodos quantitativos para o estudo da temática atesta a preocupação dos pesquisadores em criar mecanismos eficazes para investigar a empatia na realidade empírica. Ao mesmo tempo, essa predominância reforça as lacunas sobre os processos de desenvolvimento da empatia e sua manutenção, bem como, sobre os fatores sociais que interferem na empatia profissional e sobre os impactos dos contextos das instituições de saúde na empatia do profissional em formação.

$\mathrm{Na}$ coleta de dados, há predomínio das escalas psicométricas como instrumento de pesquisa, para quantificar um escore para aferir 
níveis de empatia dos profissionais. O uso de escalas para mensurar a empatia dos sujeitos participantes aparece como meio de acesso exclusivo aos dados de pesquisa em 32 (96,96\%) dos estudos, excetuando-se o estudo qualitativofenomenológico ${ }^{(36)}$ e um estudo que analisou a empatia de crianças também por meio de observação ${ }^{(37)}$. A utilização de escalas construídas pelos próprios autores também apareceu nos estudos analisados nesta revisão ${ }^{(9-10,34,38)}$.

Não há consenso entre os pesquisadores sobre como mensurar a ocorrência da empatia nos profissionais, e justifica-se esse fato, não apenas pelas diferentes conceituações que o fenômeno recebe no campo da saúde, mas, também, em razão das diferenças entre as profissões. Como exemplo, estudo cita que os instrumentos de mensuração da empatia para psicólogos e médicos não pode ser semelhante ao do enfermeiro, pois os dois primeiros profissionais têm características consultoriais (atendimentos em consultórios) no encontro com os pacientes e a enfermagem não possui tal característica $^{(31)}$. Contudo, atualmente, com o desenvolvimento da profissão de enfermagem em diferentes espaços de atuação, como nos serviços de saúde da atenção primária, a enfermagem também vem adquirindo essa característica.

As escalas para medir a empatia em estudos da área da psicologia são variadas, com destaque na amostra aqui analisada para a Empathic Understanding Scale de Carkhuff ${ }^{(18)}$, usada em vários estudos. A escala Interpersonal Reactivity Index (IRI) surge como um instrumento para medir a empatia e a simpatia. Essa escala é composta por 28 itens, cada item com 5 opções do tipo Likert. O escore da escala varia de 7 a 28 , sendo que quanto maior o escore obtido, mais empático é o sujeito que a responde ${ }^{(29)}$. Outra escala é a Consultation and Relational Empathy (CARE), que surge nos achados desta pesquisa, sendo utilizada nos estudos médicos no contexto das consultas na atenção primária. Na amostra de estudos da presente revisão ela aparece sendo validada para o uso na enfermagem ${ }^{(39)}$. Ademais, a escala CARE possui uma versão brasileira validada para medir a empatia de enfermeiros ${ }^{(40)}$.

Já a Jefferson Scale of Empathy (JSE) foi utilizada em 02 (6,06\%) dos estudos. Ela contém 20 itens, com escala de medida tipo Likert de 7 pontos. A JSE já foi traduzida até o momento para 39 línguas e é frequentemente utilizada em estudos médicos no EUA ${ }^{(29)}$. No Brasil, já foi utilizada para verificar os níveis de empatia de estudantes de medicina, em pesquisa que discute a importância da empatia em meio as virtudes da profissão médica ${ }^{(41)}$.

Estudos da área da psicologia e medicina, predominantes em relação ao tema empatia, tem por característica o emprego de escalas para mensurar a empatia de profissionais e estudantes. A enfermagem, apesar de representar uma parcela menor de pesquisas em nossa amostra, foi a única área a apresentar um estudo qualitativo sobre o tema. É evidenciada uma lacuna em investigações com delineamento qualitativo e abordagem compreensiva. A aplicação da abordagem qualitativa, na pesquisa com a temática empatia, é útil, pois permite o aprofundamento dos aspectos da subjetividade que perpassa essa questão, já que somente sua quantificação ou descrição não seriam suficientes para a explicação do fenômeno. Além disso, a integração de ambas as abordagens consolida arcabouço teórico de maior densidade e com evidências científicas contundentes.

Observa-se que a maioria dos estudos aqui analisados utilizou programas estatísticos para analisar seus dados. O único estudo qualitativo em nossa amostra analisou os dados seguindo a fenomenologia descritiva ${ }^{(36)}$.

\section{Resultados obtidos nos estudos sobre empatia em saúde}

A tônica dos resultados obtidos nas pesquisas sobre empatia é dada pela preocupação em desenvolver e validar instrumentos e justifica o investimento em estudos quantitativos, com o objetivo de mensurar níveis psicométricos de empatia e o controle desses níveis em diferentes situações no campo da saúde, tal como por meio de treinamentos para o desenvolvimento da empatia. Há necessidade de se investigar mais sobre como esses níveis de empatia se desenvolvem nos profissionais. Porém, ao medirem a empatia por meio de questionários psicométricos e escalas do tipo Likert, os estudos deixam de compreender os processos pelos quais ela se desenvolve e se mantém nos profissionais, ao longo do tempo de formação e atuação.

Dentre os fatores sociais que influenciam os níveis de empatia está o sexo do profissional. As estudantes de medicina apresentam maiores acertos em reconhecer a expressão de tristeza em outras pessoas do que os estudantes homens $^{(42)}$. Assim, o gênero e a idade, tanto dos 
profissionais quanto dos pacientes, têm sido associados como influenciadores na ocorrência de empatia na saúde. No âmbito psicossocial, a idade, os desenvolvimentos psicossociais e cognitivos mostraram-se influentes na vivência de sentimentos empáticos ${ }^{(43)}$, reforçando que a idade e variáveis sociais exercem influência na empatia. Tema esse pouco explorado pelas pesquisas.

Em psicologia, a empatia é investigada quanto aos benefícios para o tratamento em relação às outras formas de abordagem. Ao mesmo tempo, são pesquisados também os efeitos da empatia sobre o aprendizado dos terapeutas. Os resultados são divergentes, ora apresentando a relevância da empatia, ora demonstrando que ela não está relacionada aos melhores resultados de atuação profissional ou aprendizagem, podendo ser substituída por outras condutas terapêuticas nos consultórios ${ }^{(18,20,44)}$.

As pesquisas que estudaram a empatia durante a formação profissional concluíram que há uma tendência à diminuição dos níveis de empatia dos estudantes, conforme se avança nos anos de estudo na graduação em medicina e enfermagem. Algumas associações de fatores que influenciam os níveis de empatia foram ressaltadas nestas pesquisas, tais como: a instituição em que estudam, o gênero e o ano do curso. Mesmo que os níveis de empatia reduzam a medida que os anos de formação aumentem, as mulheres mantém níveis mais elevados do que os homens $^{(7,45)}$. Porém, há estudo com graduando de enfermagem com escores de empatia mais elevados no terceiro ano de graduação do que no primeiro e segundo ano ${ }^{(46)}$.

Os treinamentos com simulação, em que os estudantes vivenciam como é estar na situação do paciente, apresentaram resultados positivos no aumento da empatia. A simulação ampliou a consciência dos estudantes para considerar como é a experiência de ser um paciente ${ }^{(36)}$. O mindfullness foi eficaz na diminuição do estresse e aumento da autoconfiança de alunos de graduação, mudando as reações dos alunos frente aos pacientes ${ }^{(47)}$. Treinamentos para elevar os níveis de empatia não é preocupação exclusiva de estudos com graduandos, já que treinamento em profissionais, com uso de narrativas, demonstra melhorar a empatia de médicos, enfermeiros, assistentes sociais e terapeutas no âmbito da oncologia infantil ${ }^{(48)}$.
Os estudos sugerem a existência de vários fatores ligados aos profissionais e aos pacientes que interferem no nível de empatia na relação entre ambos. Pacientes mulheres demonstram suas emoções com maior intensidade do que os homens. Nas oportunidades empáticas, as médicas mulheres, dão respostas empáticas com maior frequência do que os homens ${ }^{(49)}$. No âmbito da empatia, já se comprovou que as mulheres enfermeiras possuem maior capacidade de tomada de perspectiva e compreensão dos sentimentos dos pacientes do que os homens ${ }^{(50)}$.

Há fatores que predizem a percepção dos pacientes sobre a empatia do profissional. Pacientes mais velhos ou com piores condições de saúde tendem a dar mais importância para a empatia em comparação com os pacientes mais jovens ou com melhores condições de saúde ${ }^{(39)}$.

No âmbito infantil, crianças com condições crônicas respondem de modo mais empático à interação com profissionais do que crianças saudáveis ${ }^{(51)}$. Assim, fatores ligados ao profissional que contribuem para que a empatia seja percebida pelos pacientes são: sexo feminino, ser conhecido pelo paciente, resguardar a privacidade durante a consulta, discutir o tratamento, usar a comunicação não verbal e demonstrar competência técnica ${ }^{(9)}$.

$\mathrm{Na}$ assistência hospitalar, a equipe de saúde perde de um a dois terços das oportunidades de se comunicar empaticamente com os cuidadores de familiares hospitalizados ${ }^{(8)}$. Especificamente sobre os profissionais de enfermagem, foi encontrado que em $75 \%$ das oportunidades em que os pacientes demonstraram emoção, os enfermeiros responderam com encorajamento mínimo e reagiram com maior grau de reconhecimento do paciente quando as emoções demonstradas por estes eram negativas ${ }^{(52)}$.

Na Alemanha, foi evidenciado que ater-se apenas ao aspecto verbal da comunicação não é suficiente para avaliar a empatia na interação entre profissional e paciente, e que aspectos não verbais como voz e visão participam da comunicação empática ${ }^{(53)}$.

$\mathrm{O}$ desenvolvimento de instrumentos e suas validações foi foco de algumas investigações. A psicologia apresenta variadas formas de medida da empatia. Nos estudos aqui analisados elas são representadas por escalas tais como a Empatic Understanding Scale de Carkhuff e Group Assessment of Interpersonal Traits - GAIT ${ }^{(18)}$, ou 
Traux e Barret-Lennard ${ }^{(44)} ; \quad$ Empathic Understanding Scale que conseguiu aferir a elevação dos níveis de empatia de enfermeiros psiquiátricos e estudantes de enfermagem ${ }^{(19)}$. Os instrumentos Interpersonal Reactivity Index (IRI), usado para medir a empatia e a simpatia, e a Jefferson Scale of Empathy (JSE), desenvolvido para medir a empatia no contexto dos cuidados médicos, mostraram-se acurados na verificação dos níveis de empatia em estudantes de medicina $^{(29)}$.

\section{CONCLUSÕES}

A empatia tem sido considerada como objeto de investigações científicas em saúde, e seus benefícios foram descritos em diferentes estudos. É um tema reemergente em pesquisas que tratam da relação profissional-paciente em distintas áreas da saúde, com destaque das pesquisas iniciais na psicologia, que se expandiram para área médica e de enfermagem.

O debate sobre a ocorrência da empatia ainda está em pleno desenvolvimento, especialmente quando se observam as diferentes explicações para sua ocorrência, que perpassam campos da cognição, emoção e atividade neuromotora. A validação de escalas para medir seus níveis, demonstra um "estado da arte" da produção científica que ainda intenta criar instrumentos para investigar esse objeto de pesquisa de modo objetivo e mensurável. Porém, muitas pesquisas sobre empatia evidenciam a necessidade de estudos qualitativos para investigação de aspectos que se supõem, estejam influenciando a empatia, como as características sociais dos participantes. Além de pesquisas quantitativas que validem os resultados já encontrados em outros contextos de serviços de saúde, com amostragens de participantes mais amplas. O destaque é para a empatia associada ao sexo feminino, recorrentemente mais alta nas diferentes pesquisas sobre o tema, em díspares cenários de estudos primários, uma associação ainda não explicada pela literatura.

A empatia é um forte constructo capaz de trazer à tona a discussão sobre a relação profissional-paciente e torná-la acessível por meio de investigações científicas. Foi constatada, contudo, a escassez de pesquisas brasileiras sobre empatia no campo da saúde, mesmo que este tema já tenha sido pesquisado de modo específico em diferentes regiões do mundo. Contudo, entre a produção e publicação desta pesquisa, considera-se que publicações recentes possam já surgir como parte de uma produção brasileira, tendo como objeto principal de estudo a empatia em saúde, haja vista a crescente produção científica sobre o tema, e a curiosidade que o conceito desperta na atualidade.

As limitações deste estudo se dão pelo tratamento amplo da produção de literatura sobre a empatia. A amplitude, buscando mapear as diferentes formas que o tema aparece nas pesquisas em saúde, diminui a profundidade com que se pode tratar os dados e analisar os achados. Contudo, por ser considerado um tema reemergente na saúde, essa revisão priorizou o mapeamento mais extenso dele, com menor profundidade de análise das evidências. Sugeremse novas pesquisas, tais como revisões sistemáticas com avaliação dos níveis de evidências em pesquisa em cada área do conhecimento, enfermagem, medicina e psicologia.

Os resultados aqui encontrados podem ser utilizados na prática acadêmica para subsidiar novas pesquisas sobre o tema da empatia, principalmente no contexto brasileiro, no qual há pouca produção sobre o tema, contribuindo para o indicativo de novos estudos e auxiliando os pesquisadores que se iniciam na temática.

\section{REFERÊNCIAS}

1- Mayernyik MA, Oliveira FAG. O cuidado empático: contribuições para a ética e sua interface com a educação moral na formação em saúde. Rev Bras Educ Med. 2016;40(1):11-20. DOI: 10.1590/1981-52712015v40n1e01752015

2- Ministério da Saúde (BR). Política Nacional de Humanização (PNH). Brasília, DF: Ministério da Saúde; 2013.

3- Haddad JGV, Machado EP, Neves-Amado J, Zoboli ELCP. A comunicação terapêutica na relação enfermeiro-usuário da atenção básica: um instrumento para a promoção da saúde e cidadania. Mundo Saúde 2011 [citado em 12 jun 2018]; 35(2):145-55. Disponível em: https://www.saocamilo-sp.br/pdf/mundo saude/ 84/145-155.pdf

4- Deslandes S. Análise do discurso oficial sobre a humanização da assistência hospitalar. Ciênc Saúde Coletiva 2014;9(1):7-14. DOI: 10.1590/S1413-81232004000100002

5- Machado ER, Soares NV. Humanização em UTI: sentidos e significados sob a ótica da equipe de saúde. R Enferm Cent O Min. 2016;6(3):2342-8. DOI: $10.19175 /$ recom.v6i3.1011 
6- Carli BS, Ubessi LD, Pettenon MK, Righi LB, Jardim VMR, Stumm EMF. The humanization theme in intensive care in health studies. J Res Fundam Care 2018;10(2):326-33. DOI: 10.9789/2175-5361.2018.v10i2.326-333

7- Narvaéz VPD, Palacio LMA, Caro SE, Silva MG, Castilho JÁ, Bilbao JL et al. Orientación empática de estudiantes de medicina en tres universidades de Barranquilla, Colombia y en una universidad de República Dominicana. Arch Argent Pediatr. 2014;112(1):41-9. DOI: 10.5546/aap.2014.e41

8- Wittenberg-Lyles E, Debra PO, Demiris G, Rankin A, Shaufield S, Kruse RL. Conveying empathy to hospice family caregivers: team responses to caregiver empathic communication. Patient Educ Couns. 2012;89(1):31-7. DOI: 10.1016/j.pec.2012.04.004

9- Birhanu Z, Tsion A, Mirkuzie W, Sudhakar M. Predictors of perceived empathy among patients visiting primary health-care centers in central Ethiopia. Int J Qual Health Care 2012;24(2):161-8. DOI: $10.1093 /$ intqhc/mzs001

10- Soltner C, Giquello JÁ, Monrigal-Martin C, Beydon L. Continuous care and empathic anaesthesiologist attitude in the preoperative period: impact on patient anxiety and satisfaction. $\mathrm{Br} J$ Anaesth. 2011;106(5):680-6. DOI: $\underline{10.1093 / \text { bja/aer034 }}$

11- Moghaddasian S, Dizaji SL, Mahmoudi M. Nurses empathy and family needs in the Intensive Care Units. J Caring Sci. 2013;2(3):197-201. DOI: 10.5681/jcs.2013.024

12- Reynolds WJ, Scott BS. Do nurses and other professional helpers normally display much empathy? J Adv Nurs. 2000;31(1):226-34. DOI: 10.1046/j.1365-2648.2000.01242.x

13- Kestenberg CCF. A habilidade empática é socialmente aprendida: um estudo experimental com graduandos de enfermagem. Rev Enferm UERJ 2013 [citado em 15 jan 2018]; 21(4):427-33. Disponível em: http://www.facenf.uerj.br/v21n4/v21n4a02.pdf

14- López MB, Filippetti VA, Richaud MC. Empatía: desde la percepción automática hasta los procesos controlados. Av Piscol Latinoam. 2014 [citado em 2019 ago 05]; 32(1):37-51. Disponível

em:

http://www.scielo.org.co/pdf/apl/v32n1/v32n1a0 4.pdf

15- Whittemore $R$, Knafl $K$. The integrative review: updated methodology. J Adv Nurs. 2005;52(5):546-53. DOI: 10.1111/j.13652648.2005.03621.x
16- University of Toronto. Searching the literature: a guide to comprehensive searching in the health sciences. Toronto: University of Toronto; 2019. [citado 2019 ago 05]. Disponível em:

https://guides.library.utoronto.ca/c.php?g=57791 9\& $=4304403$

17- Moher D, Liberati A, Tetzlaff J, Altman DG. Preferred reporting items for systematic reviews and meta-analyses: the PRISMA Statement. PLoS Med. 2009;6(7):e1000097.

DOI:

10.1371/journal.pmed.1000097

18- Dooley D, Lange AJ, Whiteley JM. Sources of discrepancy in Carkhuff and GAIT measurements of empathy. Psychother Theor Res Pract. 1979; 16(3):337:44. DOI: 10.1037/h0085899

19- Nagano H. Empathic understanding: constructing an evaluation scale from the microcounseling approach. Nurs Health Sci. 2000; 2(1):17-2. DOI: $10.1046 / \mathrm{j} \cdot 1442-$ 2018.2000.00035.x.

20- Esse JT, Wilkins W. Empathy and imagery in avoidance behavior reduction. J Consult Clin Psychol. 1978;46(1):202-3. DOI: 10.1037/0022006X.46.1.202

21- Kimberlin C, Friesen D. Effects of client ambivalence, trainee conceptual level, and empathy training condition on empathic responding. J Couns Psychol. 1977;24(4):354-8. DOI: $10.1037 / 0022-0167.24 .4 .354$

22- Perry MA. Modeling and instructions in training for counselor empathy. J Couns Psychol. 1975;22(3):173-9. DOI: 10.1037/h0076713

23- Dalton RF, Sundblad LM. An application of principles of social learning to training in communication of empathy. J Couns Psychol. 1973;20(4):378-83. DOI: $10.1037 /$ h0034789

24- Heck EJ, Davis CS. Differential expression of empathy in a counseling analogue. J Couns Psychol. 1973;20(2):101-4. DOI: 10.1037/h0034171

25- Payne PA, Weiss SD, Kapp RA. Didactic, experiential, and modeling factors in the learning of empathy. J Couns Psychol. 1972;19(5):425-9. DOI: 10.1037/h0033193

26- Kurtz RR, Grummon DL. Different approaches to the measurement of therapist empathy and their relationship to therapy outcomes. J Consult Clin Psychol. 1972;39(1):1065. DOI: $10.1037 /$ h0033190

27- Bachrach H, Mintz J, Luborsky L. On rating empathy and other psychotherapy variables: an experience with the effects of training. J Consult 
Clin Psychol. 1971;36(3):445. DOI: 10.1037/h0031109

28- Fish JM. Empathy and the reported emotional experiences of beginning psychotherapists. J Consult Clin Psychol. 1970;35(1):64-9. DOI: 10.1037/h0029630

29- Hojat M, Spandorfer J, Louis DZ, Gonnela JS. Empathic and sympathetic orientations toward patient care: conceptualization, measurement, and psychometrics. Acad Med. 2011;86(8):98995. DOI: 10.1097/ACM.0b013e31822203d8

30- Rogers CR. The necessary and sufficient conditions of therapeutic personality change. J Consult Clin Psychol. 1957;21(2):95-103. DOI: http://dx.doi.org/10.1037/h0045357

31- La Monica EL. Construct validity Construct vahdity of an empathy instrument. Res Nurs Health 1981; 4(4):389-400. DOI: 10.1002/nur.4770040406

32- Kuremyr D, Kihlgren M, Norberg A, Astrom $\mathrm{S}$, Karlsson I. Emotional experiences, empathy and burnout among staff caring for demented patients at a collective living unit and a nursing home. J Adv Nurs. 1994;19(4):670-79. DOI: 10.1111/j.1365-2648.1994.tb01137.x

33- Blair RJR. Responding to the emotions of others: dissociating forms of empathy through the study of typical and psychiatric populations. Conscious Cogn. 2005;14(4):698-718. DOI: 10.1016/i.concog.2005.06.004

34- Seehausen M, Kazzer P, Bajbouj M, Heekeren AMJ, Klann-Delius G, Menninghaus $W$ et al. Effects of empathic social responses on the emotions of the recipient. Brain Cogn. 2016; 103:50-61. DOI: 10.1016/i.bandc.2015.11.004

35- López MB, Filippetti VA, Richaud MC. Empatía: desde la percepción automática hasta los procesos controlados. Av Piscol Latinonoam. 2014;32(1):37-51. DOI: 10.12804/apl32.1.2014.03 36- Díaz DA, Maruca AT, Kuhnly JE, Jeffries $P$, Grabon N. Creating caring end empathic nurses: a simulated ostomate. Clin Simul Nurs. 2015; 11(12):513-8. DOI: 10.1016/i.ecns.2015.10.002

37- Fernald CD, Corry JF. Empathic versus directive preparation of children for needles. Child Health Care 1981;10(2):44-7. DOI: 10.1207/s15326888chc1002 1

38- Kondo K, Fujimori $M$, Shirai $Y$, Yamada $Y$, Ogawa $A$, Hizawa $N$, et al. Characteristics associated with empathic behavior in Japanese oncologists. Patient Educ Couns. 2013;93(2):350-3. DOI: 10.1016/j.pec.2013.06.023

39- Bikker AP, Fitzpatrick B, Murphy D, Mercer SW. Measuring empathic, person-centred communication in primary care nurses: validity and reliability of the Consultation and Relational Empathy (CARE) Measure. BMC Fam Pract. 2015; 16:149. DOI: $10.1186 / \mathrm{s} 12875-015-0374-y$

40- Savieto RM, Mercer S, Matos CCP, Leão ER. Enfermeiros na triagem no serviço de emergência: autocompaixão e empatia. Rev. Latino-Am Enfermagem 2019;27:1-11. DOI: 10.1590/1518-8345.3049.3151

41- Schweller M, Ribeiro DL, Celeri EC, Filho $M A C$. Nurturing virtues of the medical profession: does it enhance medical students' empathy? Int J Med Educ. 2017;8:262-7. DOI: 10.5116/ijme.5951.6044

42- Ávila RF, Morais D, Bomfim AJ, Chagas MHN. Empatia e reconhecimento de expressões faciais de emoções básicas e complexas em estudantes de Medicina. J Bras Psiquiatr 2016;65(3):209-14. DOI: 10.1590/0047-2085000000126

43- Sampaio LR, Moura MAR, Guimarães PRB, Santana LB, Camino CPS. Sentimentos empáticos em crianças, adolescentes e adultos. Psic: teor e pesq. 2013;29(4):393-401. DOI: 10.1590/S0102$\underline{37722013000400005}$

44- Payne PA, Gralinski DM. Effects of Supervisor style and empathy upon counselor learning. J Couns Psychol. 1968;15(6):517-21. DOI: 10.1037/h0026255

45- Özakgül AA, Sendir M, Atav AS, Kiziltan B. Attitudes towards HIV/AIDS patients and empathic tendencies: a study of Turkish undergraduate nursing students. Nurse Educ Today 2014;34(6):929-33. DOI: 10.1016/j.nedt.2013.10.018

46- Lauder W, Reynolds W, Smith A, Sharkey S. A comparison of therapeutic commitment, role support, role competency and empathy in three cohorts of nursing students. J Psychiatr Ment Health Nurs. 2002;9(4):483-91. DOI: 10.1046/j.1365-2850.2002.00510.x

47- Beddoe AE, Murphy SO. Does mindfulness decrease stress and foster empathy among nursing students? J Nurs Educ. 2004 [citado em 2019 ago 05];43(7):305-12. Disponível em: https://www.ncbi.nlm.nih.gov/pubmed/15303583 48- Stephen AS, Stanley P, Charon R. Pediatric narrative oncology: interprofessional training to promote empathy, build teams, and prevent burnout. J Support Oncol. 2008 [citado em 2019 ago 05]; 6(7):307-12. Disponível em: https://www.ncbi.nlm.nih.gov/pubmed/18847073 49- Bylund CL, Makoulc G. Empathic communication and gender in the physicianpatient encounter. Patient Educ Couns. 2002; 
48(3):207-16. DOI: $\quad \underline{10.1016 / 50738-}$ 3991(02)00173-8

50- Giménez-Espert MC, Prado-Gascó VJ. The moderator effect of sex on attitude toward communication, emotional intelligence, and empathy in the nursing field. Rev Latino-Am Enfermagem 2017;25:1-7. DOI: 10.1590/1518$\underline{8345.2018 .2969}$

51- Sterling CM, Friedman AG. Empathic responding in children with a chronic illness. Chindren's Health Care 1996;25(1):53-69. DOI: 10.1207/s15326888chc2501 5

52- Eide $H$, Sibbern $T$, Johannessen T. Empathic accuracy of nurses' immediate responses to fibromyalgia patients' expressions of negative emotions: an evaluation using interaction analysis. J Adv Nurs. 2011;67(6):1242-53. DOI: 10.1111/j.1365-2648.2010.05579.x

53- Nicolai J, Demmel R, Farsh K. Effects of mode of presentation on ratings of empathic communication in medical interviews. Patient Educ Couns. 2010;80(1):76-9. DOI: 10.1016/j.pec.2009.10.014

Nota: Este artigo é resultante da disciplina "Introdução à busca em bases de dados" do Programa de Pós-Graduação em Enfermagem da Universidade Federal de Mato Grosso.

Recebido em: 11/04/2018

Aprovado em: 14/08/2019

Endereço de correspondência:

Leandro Felipe Mufato

Rua 50. N. 172.

Condomínio Mônaco. Apto 102.

Bairro: Jardim Europa.

CEP: 780300-000 - Tangará da Serra/MT - Brasil

E- mail: leandro.mufato@gmail.com 